\title{
Determining Optical Constants of Selenium Thin Films using the Envelope Method
}

\author{
${ }^{1}$ Nehad M. Tashtoush and ${ }^{2}$ Osamah ALkasassbeh \\ ${ }^{1}$ Department of Physics, Faculty of Science, Yarmouk University, Irbid, Jordan \\ ${ }^{2}$ Department of Physics, Faculty of Science, Mutah University, Al-Karak, Jordan
}

Received 2012-05-02, Revised 2012-07-05; Accepted 2013-04-11

\begin{abstract}
Two Selenium thin films were deposited on glass substrates using thermal evaporation technique. The optical constants (refractive index, absorption coefficient and extinction coefficient and energy gap) were calculated using the Transmittance (T) spectrums of the films in the spectral range of 500-900 nm. The envelope method was used to determine optical constants. The calculated refractive index was found to be in the range of 2.60 to 2.85 and the Energy gap $\left(\mathrm{E}_{\mathrm{g}}\right)$ was found to be $1.8 \mathrm{eV}$ which are in agreement with other studies made using other procedures. The films were found to be amorphous according to the results obtained by XRD technique. This method can be used to have a good results for many tranparent thin films with more mathematical tools.
\end{abstract}

Keywords: Refractive Index, Extinction Coefficient, Optical Constants, Absorption Coefficient, Amorphous Selenium, Envelope Method

\section{INTRODUCTION}

Selenium (Se) thin films and its other copounds such as CuInSe are used in many industrial applications such as photovoltaic cell and optical devices industry (Bhadra et al., 1999; Bindu et al., 2002). Recently, the optical and electrical properties of a-Se films have a substantial renewed interest due to its use as an ultra-sensitive photoconductor in newly developed flat panel x-ray image detector and high definition digital and video camera (Chong, 2006). Moreover, doping Se with other metals like Te will enhance its optical properties, such as decreasing the energy band gap, made it of much interest for applications in new technology (Zishan et al., 2012). The optical constants are of much importance for applications. These optical constants are the refractive index (n), the extinction coefficient $(\mathrm{k})$ which is a measure of the energy loss in material and is related to the absorption coefficient $(\alpha)$ according to the relation Equation 1:

$\mathrm{k}=\frac{\alpha \lambda}{4 \pi}$ where, $\lambda$ is the wavelength of the incident light (Marquez et al., 1992; Chavez-Rivas et al., 1982). The importance of the absorption coefficient is that it indicates how readily photons will be absorbed by the material.

In order to measure the optical constants, some experimental measurements and data must be known and measured. This can be accomplished by measuring the Reflectance (R) and/or Transmittance (T), of the film; then solving three non-linear equations can give the optical constants (Heavens, 1955). However, solving these equations for many semiconducting materials is not quite easy. Therefore, an other method based on the ability of the film to form an interference pattern on $\mathrm{T}$ spectrum and then using what is referred to as the envelope method is an alternative easy method which was proposed by (Swanepoel, 1983).

Optical constants of Se thin films have been investigated and calculated in many ways but not the envelope method. Therefore, this method will be used to measure the optical constants for two different Se thin film and comparing the results with what have been already published (Salwan et al., 1990; Bindu et al., 2002; Bhadra et al., 1999; Gross et al., 1977; Corresponding Author: Nehad M. Tashtoush, Department of Physics, Faculty of Science, Yarmouk University, Irbid, Jordan 
Manifacier et al., 1976; Kasasbeh, 2010; Streetman, 1995; Tashtoush, 2002).

This method depends on the transmittance curve of the sample. In films at which the thickness of the substrate is several orders of magnitude larger than the thickness $d$ and the film is uniform, interference pattern could be seen in the VIS and IR region of the transmittance spectrum. This effect will give rise to an oscillating transmission curve. Such interference fringes will be used to determine the optical constants, as well as the film thickness. This method was completely described by Swanepoel (1983); Marquez et al. (1992) and Kasasbeh (2010).

\section{MATERIALS AND METHODS}

Selenium powder of $99.99 \%$ purity was used to prepare two samples of $a$-Se thin films using the thermal evaporation technique. This was achieved by using Edwards Coating System E306 A model. The samples were deposited on glass substrates (microscope slides) with a refractive index of about 1.52. The substrates were cleaned by the usual method of cleaning that is: using the ultrasound cleaner filled with acetone, then filled with distilled water. After this, all substrates were cleaned again with methanol and then with distilled water. Finally, a hot clean air was used to dry the substrates.

The base pressure inside the chamber was less than $10^{-5}$ mbar. The film thickness was controlled and monitored using a Quartz crystal thickness monitor of the type Edwards FTM5 model.

The Transmittances $(T)$ of the films were measured over the range of 450-900 nm wavelength using a UV-VIS spectrophotometer, Specord M500 model.

\section{RESULTS AND DISCUSSION}

Many experiments have been conducted to study the optical properties of $a$-Se thin films. Figure 1 shows a typical XRD pattern for one of the as deposited Se thin films. The pattern shows that the film was amorphous.

Figure 2 shows a typical transmittance spectrum (the solid line) of a-Se thin film of a thickness of 1580 $\mathrm{nm}$. It seems that there is no transmission (high absorption) in the range of $300 \mathrm{~nm}$ to about $580 \mathrm{~nm}$. The curve shows an interference fringes on the high transmittance (low absorption) region of wavelength. For measurements needs, the curve can be split into two regions; the first region is the strong and medium absorption region which appears in the interval 550-700 $\mathrm{nm}$, the second region appears in the interval of 700-900 $\mathrm{nm}$ and known as the transparent region.
It is important to mention that the photons with energies less than band gap are transmitted, on the other hand the photons with energies greater than or equal to band gap can be absorbed, where a photon with energy less than $E_{g}$ is unable to excite an electron from the valance band to the conduction band, thus in a pure semiconductor there is a negligible absorption of photons with $h v<E_{g}$. This explains why some materials are transparent, like our sample, in certain wavelength range (Streetman, 1995).

In order to determine the optical properties we have to build up two curves that are $T_{M}$ curve which is related to the maximum points at which the interference took place and $T_{m}$ curve which is related to the points at which minimum points at which the interference took place (dashed line on Fig. 2. $T_{M}$ and $T_{m}$ are considered to be continuous function of $\lambda$. Also, the refractive index is a function of $\lambda$, i.e., $n(\lambda)$. The two curves of $T_{M}$ and $T_{m}$ formed the envelope of the T curve as shown in Fig. 2. These curves are constructed using three point extrapolation method. As it is indicated on Fig. 2, we see that at any $\lambda$, for any value of $T_{M}$ there is a corresponding value of $\mathrm{T}_{\mathrm{m}}$.

One of the advantages of this method is that it can be used to measure the refractive index of the substrate (s). This is by using the maximum transmittance $\left(\mathrm{T}_{\mathrm{M}}\right)$ value in the second region of spectrum in Fig. 2, then the equation (Swanepoel, 1983; Marquez et al., 1992) Equation 2:

$$
\mathrm{s}=\frac{1}{\mathrm{~T}_{\mathrm{M}}}+\sqrt{\frac{1}{\mathrm{~T}_{\mathrm{M}}^{2}}-1}
$$

The refractive index of the substrate was measured and found to be 1.52 .

Using the data of the first region of the curve in Fig. 2, the refractive index can be calculated using the following equation (Equation 3 and 4) (Swanepoel, 1983; Marquez et al., 1992):

$$
\mathrm{n}=\sqrt{\mathrm{N}+\sqrt{\mathrm{N}^{2}-\mathrm{s}^{2}}}
$$

Where:

$$
\mathrm{N}=2 \mathrm{~s}\left(\frac{\mathrm{T}_{\mathrm{M}}-\mathrm{T}_{\mathrm{m}}}{\mathrm{T}_{\mathrm{M}} \mathrm{T}_{\mathrm{m}}}\right)+\frac{\mathrm{s}^{2}+1}{2}
$$

Figure 3 shows the calculated values of refractive index for the as deposited Se thin film as a function of wavelength. As Fig. 3 shows, the refractive index decreases from $2.815-2.615$ as $\lambda$ increases from $632-890$ 
$\mathrm{nm}$ (the dot points in the figure). The calculated values of $n(\lambda)$ can be fitted to a reasonable function such as the two-terms Cauchy dispersion relationship, $n(\lambda)=a+\frac{b}{\lambda^{2}}$, which can be used for extrapolation to shorter and longer wavelengths. The fit of the values of $n(\lambda)$ yields $n(\lambda)=2.385+\frac{168385}{\lambda^{2}}$. The full curve in Fig. 3 represents the fitting curve while the dot points are the calculated ones.

In order to measure the absorption coefficient $(\alpha)$ of the sample the following equation was used (Equation 5) (Swanepoel, 1983; Marquez et al., 1992):

$\alpha=\frac{-\ln [\mathrm{x}]}{\mathrm{d}}$ where, $\mathrm{d}$ is the film thickness and $x$ is given by Equation 6 and 7 :

$$
x=\frac{E_{m}-\sqrt{E_{m}{ }^{2}-\left(n^{2}-1\right)^{3}\left(n^{2}-s^{4}\right)}}{(n-1)^{3}\left(n-s^{2}\right)}
$$

where $E_{m}$ is given by:

$$
E_{m}=\frac{8 n^{2} s}{T_{M}}+\left(n^{2}-1\right)\left(n^{2}-s^{2}\right)
$$

The measured values of the absorption coefficient, $(\alpha)$, as a function of the wavelength is shown in Fig. 4

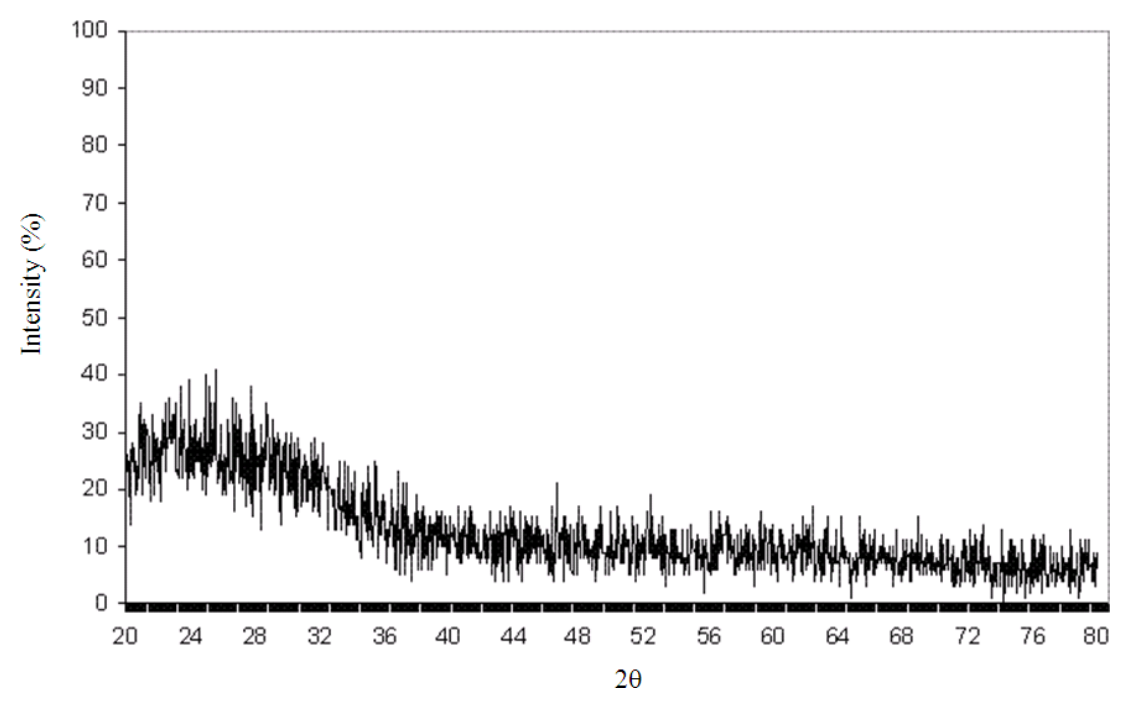

Fig. 1. The X-ray diffraction pattern of as deposited Se thin film on glass substrate

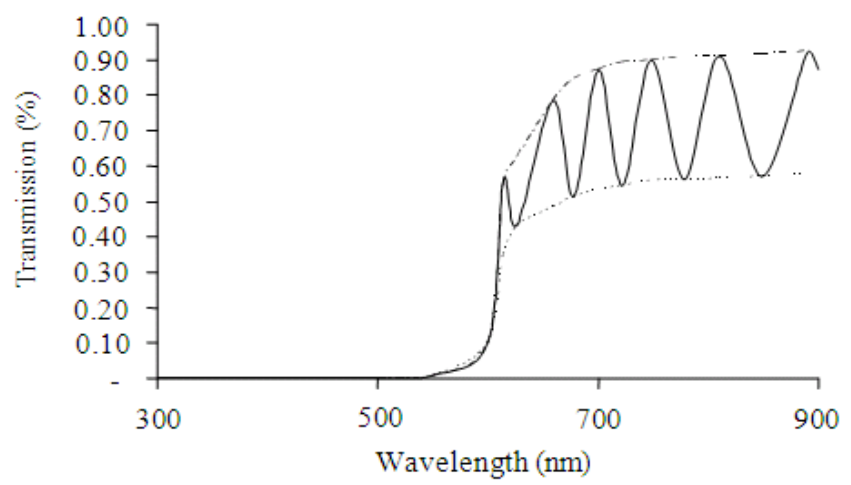

Fig. 2. The transmission spectrum (full curve) for a $1580 \mathrm{~nm}$ a-Se thin film on a glass substrate 
Nehad M. Tashtoush and Osamah ALkasassbeh / American Journal of Applied Sciences, 10 (2): 164-171, 2013

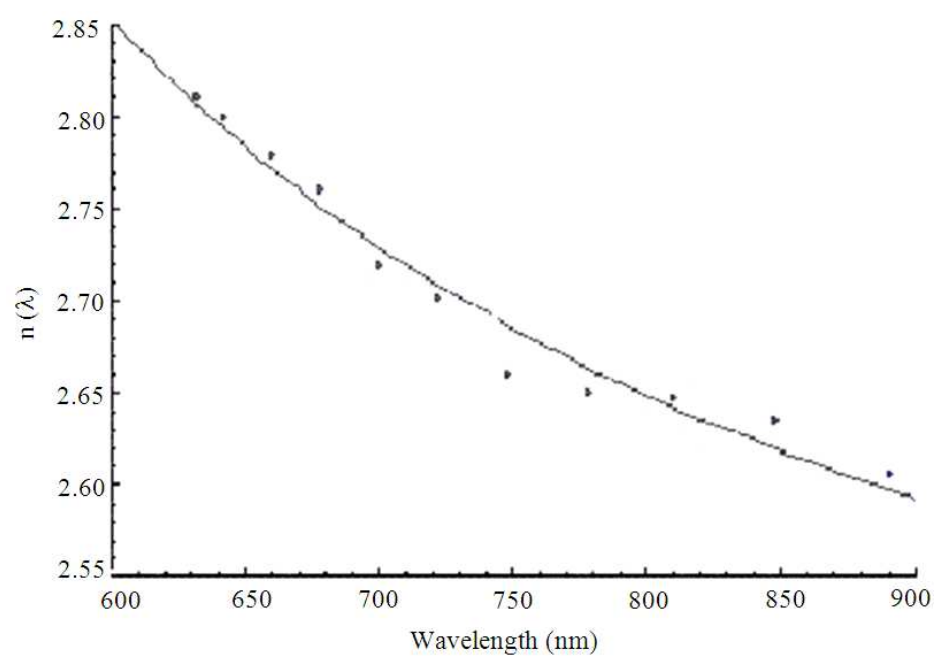

Fig. 3. Refractive index of as deposited Se $(1580 \mathrm{~nm})$ film versus wavelength

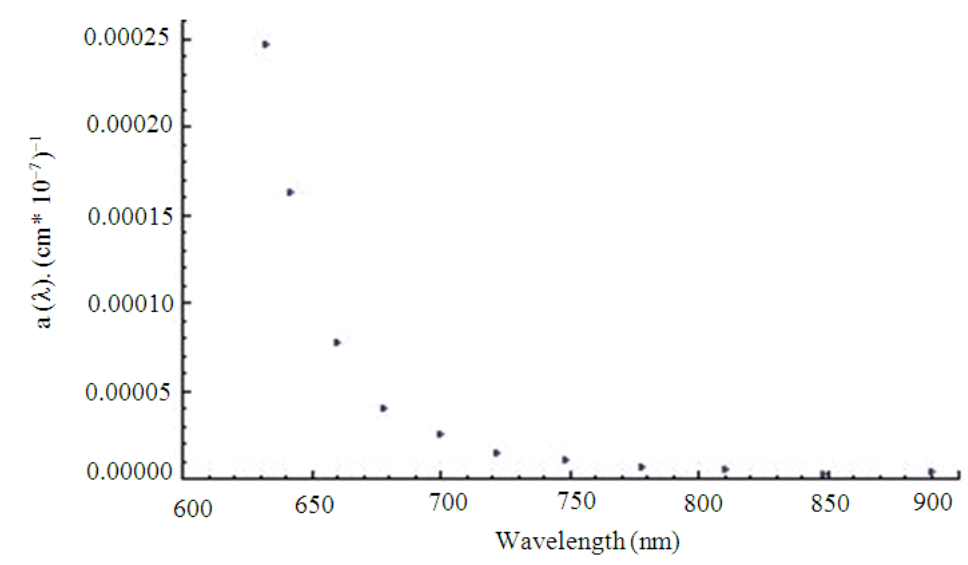

Fig. 4. Absorption coefficient of the as deposited Se $(1580 \mathrm{~nm})$ thin film versus wavelength

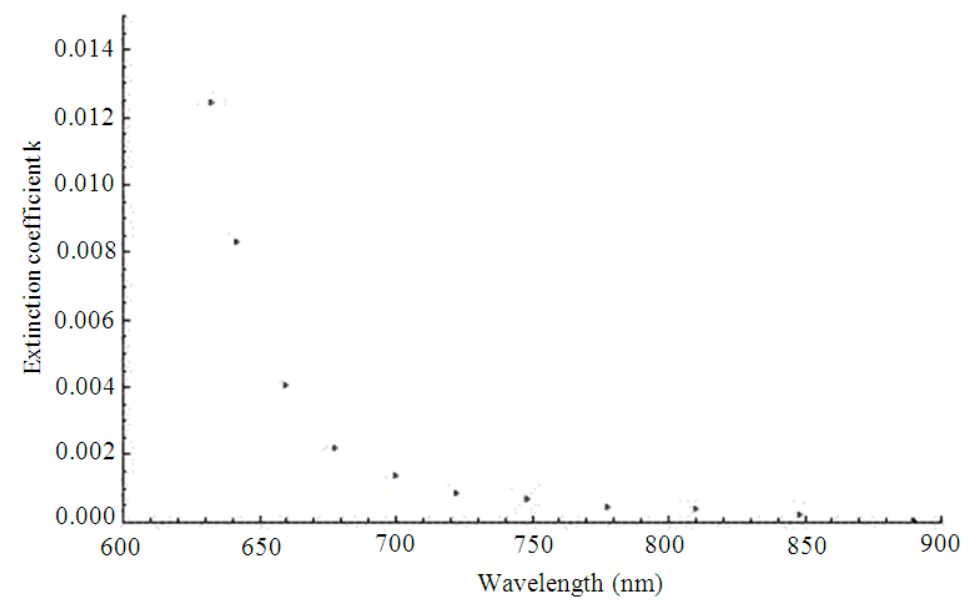

Fig. 5. Extinction coefficient of as deposited Se $(1580 \mathrm{~nm})$ thin film versus wavelength 
Nehad M. Tashtoush and Osamah ALkasassbeh / American Journal of Applied Sciences, 10 (2): 164-171, 2013

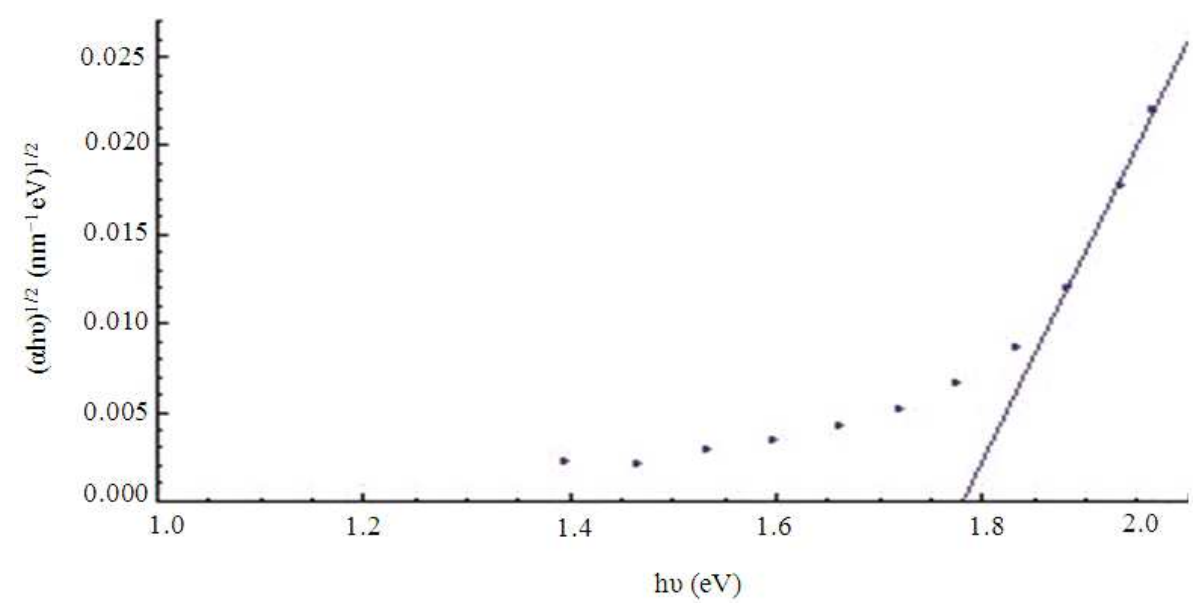

Fig. 6. $(\alpha h v)^{1 / 2}$ versus (hv) plot for $1580 \mathrm{~nm}$ Se thin film

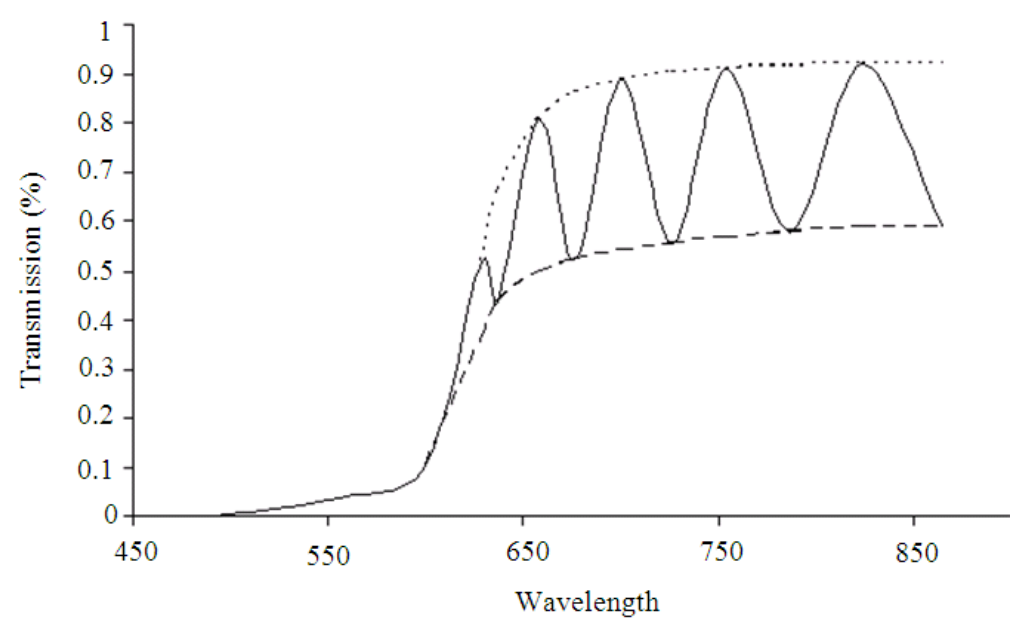

Fig. 7. The transmission spectrum (full curve) for a $1400 \mathrm{~nm}$ a-Se thin film on a glass substrate

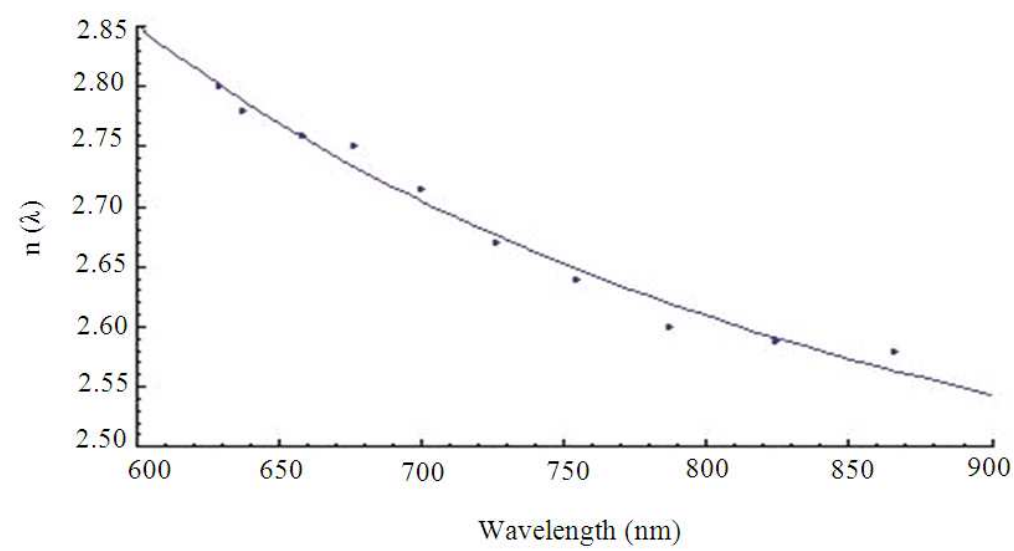

Fig. 8. Refractive index of Se (1400 nm) film against wavelength 


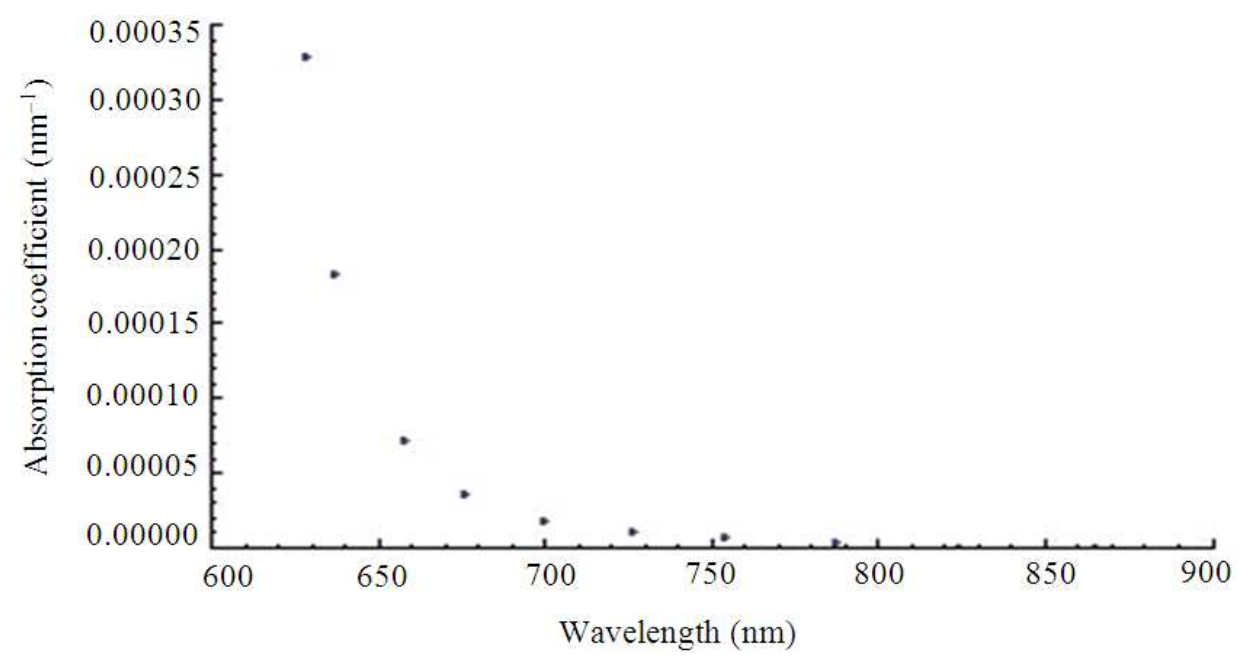

Fig. 9. Absorption coefficient of as deposited Se $(1400 \mathrm{~nm})$ thin film against wavelength

It appears that the absorption coefficient decreases from $0.00024712-0.00000379\left(\mathrm{~cm}^{*} 10^{-7}\right)^{-1}$ as wavelength increases from $632-890 \mathrm{~nm}$. Moreover, the absorption coefficient is high at the low region of wavelength while it becomes to zero (no absorption) at high wave lengths as shown on the T curve, i.e., Fig. 2.

Figure 5 shows the behavior of the extinction coefficient versus wavelength. The extension coefficient was calculated by using Equation 1. It appears that the extinction coefficient decreases from 0.012430.000026816 as wavelength increases from $632-890 \mathrm{~nm}$.

The absorption coefficient is related to the direct allowed band gap energy $\left(\mathrm{E}_{\mathrm{g}}\right)$ according to the relation (Tashtoush, 2002) Equation 8:

$\alpha h v=B\left(h v-E_{g}\right)^{N}$

where, $\mathrm{N}$ is an integer equals to 2 for amorphous Selenium.

Therefore, in order to measure the energy gap of the as deposited Se thin film, the relation between $(\alpha h v)^{1 / 2}$ and (hv) was plotted and a linear behavior is expected. Figure 6 shows this behavior. Using this curve, the energy gap of $a-S e$ thin film was calculated and found to be approximately $1.783 \mathrm{eV}$. This value is consistent with previous studies (Chong, 2006).

Another sample of $1400 \mathrm{~nm}$ thickness was prepared and the optical constants were calculated. Figure 7 shows the transmittance spectrum (solid line) as a function of wavelength. The three point extrapolation curve (dashed line) is also shown connecting between the maximum and the minimum points.

The spectrum on Fig. 7 can be classified into two regions; first region is the strong and medium absorption region which appears in the interval 550-700 $\mathrm{nm}$. The second region appears in the interval $700 \mathrm{~nm}$ to $850 \mathrm{~nm}$ which called the weak absorption region (i.e., the transparent region).

Using the same procedure used for the previous film (i.e., envelope method (Chavez-Rivas et al., 1982; Bindu et al., 2002), the refractive index was measured. The variation of refractive index as a function of wavelength is shown in Fig. 8 as a dot points. It appears that the refractive index decreases from 2.8-2.58 as wavelength increases from $628.57-865.71 \mathrm{~nm}$. The data were fitted to the two-term Cauchy dispersion relationship and the relation was found to be: $n(\lambda)=2.29812+\frac{199032}{\lambda^{2}}$. This is represented on Fig. 8 by the solid line.

The absorption coefficient $\alpha(\lambda)$ versus wavelength for the $1400 \mathrm{~nm}$ as deposited $\mathrm{Se}$ thin film is shown in Fig. 9. As the Figure shows, $\alpha(\lambda)$ decreases from $0.000328-0.00000043 \mathrm{~nm}^{-1}$ as wavelength increases from $628-865 \mathrm{~nm}$.

Figure 10 shows the behavior of the extinction coefficient as a function of wavelength. It appears that the extinction coefficient decreases from 0.016410.00003 as wavelength increases from $628-865 \mathrm{~nm}$.

In Fig. 11, $(\alpha h v)^{1 / 2}$ versus hv was plotted to determine the energy gap of the film. It was found to be about 1.815. 


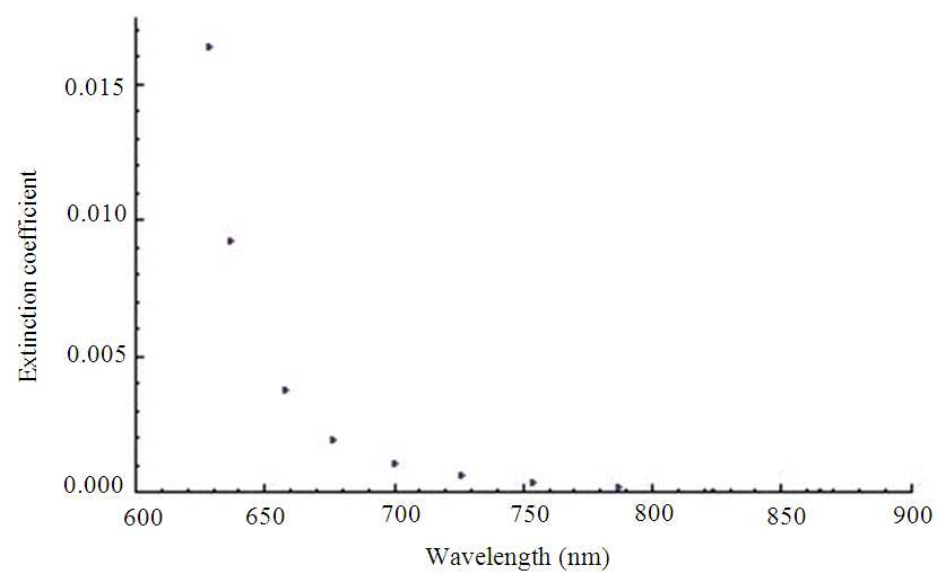

Fig. 10. Extinction coefficient of the as deposited $\mathrm{Se}(1400 \mathrm{~nm})$ thin film against wavelength

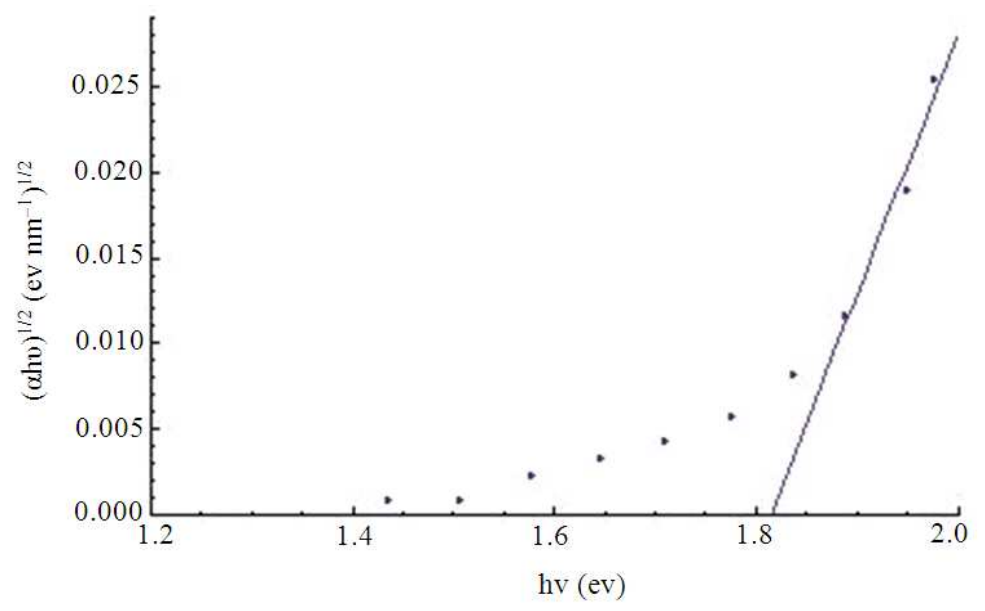

Fig. 11. $(\alpha h v)^{1 / 2}$ versus (hv) plot for $1400 \mathrm{~nm}$ a-Se thin film

\section{CONCLUSION}

In this study, the envelope method which was proposed and used by Swanepoel (1983) was used to calculate the optical constants. The refractive index was found to be in the range of 2.60 to 2.85 and the energy gap $\left(\mathrm{E}_{\mathrm{g}}\right)$ was found to be about $1.8 \mathrm{eV}$. The results were found to be in consistent with other studies that utilized other procedures. Also, the absorption coefficient and extension coefficient were found to be consistant with previous studies.

\section{ACKNOWLEDGMENT}

The researchers would like to thank Yarmouk University represented by the Deanship of graduate studies and scientific research for their kindly financial support for publishing this article.

\section{REFERENCES}

Bhadra, S.K., A.K. Maiti and K. Goswami, 1999. PhotoDarkening in GeSe film by $\mathrm{CW}$ laser irradiation. J. Mater. Sci. Lett., 18: 1543-1545. DOI: 10.1023/A:1006635610485

Bindu, K., C. Sudha, K.P. Kartha, Y. Vijayakumar and A. Kashiwaba, 2002. Structural, optical and electrical properties of $\mathrm{In}_{2} \mathrm{Se}_{3}$ thin films formed by annealing chemically deposited Se and vacuum evaporated In stack layers. Applied Surface Sci., 191: 138-147. DOI: 10.1016/S01694332(02)00172-1 
Chavez-Rivas, F., A. Ribeyron and J.M. Laurant, 1982. Optical absorption in the low frequency range of amorphous polymeric systems: Amorphous selenium and Polymethylmethacrylate. Physica, 109-110: 2119- 2121. DOI: 10.1016/03784363(82)90256-X

Chong, W.T., 2006. Optical properties of amorphous Selenium films. Master Theses, the Department of Electrical Engineering, University of Saskatchewan, Saskatchewan, Canada.

Gross, G., R.B. Stephens and D. Turnbull, 1977. On the crystallization of amorphous selenium films: Thermal effects and photoeffects. J. Applied Phys., 48: 1139-1148. DOI: $10.1063 / 1.323792$

Heavens, O.S., 1955. Optical Properties of Thin Films. 1st Edn., Dover Publications, New York, ISBN-10: 0486669246, pp: 261.

Kasasbeh, O., 2010. Optical and electrical properties of $\mathrm{Se}$ and $\mathrm{V}_{2} \mathrm{O}_{5}$ thin films. M.Sc Theses, Mutah University, Jordan.

Manifacier, J.C., J. Gasiot and J. P. Fillard, 1976. A simple method for the determination of the optical constants $\mathrm{n}, \mathrm{k}$ and the thickness of a weakly absorbing thin film. J. Phys. E: Sci. Instrum. DOI: $10.1088 / 0022-3735 / 9 / 11 / 032$
Marquez, E., J. Ramfrez-Malo, P. Villares, R. JimenezGaray and P.J.S. Ewen et al., 1992. Calculation of the thickness and optical constants of amorphous arsenic sulphide films from their transmission spectra. J. Phys. D: Applied Phys., 25: 535-535. DOI: 10.1088/0022-3727/25/3/031

Salwan, K., J. Al-Ani, Mehadi N. Al-Delaimi, H. Ali and A. Munaim et al., 1990. Optical and electrical properties of pure and doped amorphous thin selenium films. INT. J. Elect., 69: 87-95. DOI: 10.1080/00207219008920294

Streetman, B.G., 1995. Solid State Electronic Devices. 4th Edn., Prentice Hall, Englewood Cliffs, New Jeresey, ISBN-10: 0131587676, pp: 462.

Swanepoel, R., 1983. Determination of the thickness and optical constants of amorphous silicon. J. Phys. E: Sci. Instrum., 16: 1214-1222. DOI: 10.1088/00223735/16/12/023

Tashtoush, N., 2002. Variation of optical properties of RF sputtered a-Si thin films with deposition conditions. Abhath Al-Yarmouk.

Zishan, H., k. Numan, S. Sami, A. Habib and A. AlGhamdi et al., 2012. Electrical and optical properties of $\mathrm{a}-\mathrm{Se}_{\mathrm{x}} \mathrm{Te}_{100-\mathrm{x}}$ thin films. Optics Laser Technol., 44: 6-11. DOI: 10.1016/j.optlastec.2011.05.001 